\title{
LINEARLY CONTINUOUS FUNCTIONS OF FINITE AREA ${ }^{1}$
}

\author{
BY CASPER GOFFMAN
}

Communicated by A. Zygmund, June 24, 1966

1. Definitions and preliminaries. We shall consider real functions of $n$ variables with compact support.

Let $x=\left(x_{1}, \cdots, x_{n}\right)$ be a cartesian coordinate system and let $\bar{x}_{i}=\left(x_{1}, \cdots, x_{i-1}, x_{i+1}, \cdots, x_{n}\right)$ be a point in $n-1$ space, so that $x=\left(x_{i}, \bar{x}_{i}\right)$. A function $f$ is said to be of type BVT if there are equivalent functions $f_{i}, i=1, \cdots, n$, such that, for almost all $\bar{x}_{i}$, the variation $V_{i}\left(f, \bar{x}_{i}\right)$ of $f$ as a function of $x_{i}$ is finite, and $V_{i}$ is a summable function of $\bar{x}_{i}$. The functions of type BVT are those for which the partial derivatives are measures, [9], and those for which the area is finite, [1], [4]. $f$ is said to be ACT if it is BVT and the equivalent $f_{i}, i=1, \cdots, n$, may be chosen to be absolutely continuous for almost all $\bar{x}_{i}$. These are the functions whose partial derivatives are functions, [3], [11], and for which the area is given by the classical formula [4].

A function $f$ is said to be essentially linearly continuous if, for every $i=1, \cdots, n$, there is an equivalent $f_{i}$ which is continuous as a function of $x_{i}$ for almost all $\bar{x}_{i} . f$ is said to be linearly continuous if there is a $g$ equivalent to $f$ such that, for every $i=1, \cdots, n, g$ is continuous in $x_{i}$ for almost all $\bar{x}_{i}$. It is known, [5], that every essentially linearly continuous function which is of type BVT is linearly continuous, and that in the case of functions of two variables these are the ones for which the area is equal to the Hausdorff 2 dimensional measure of the graph. Linearly continuous functions which are of type BVT will be called of type $L$.

Let $f$ be of type BVT, let $\left(\mu_{1}, \cdots, \mu_{n}\right)$ be its gradient measure, and let $m$ be Lebesgue measure. The total variation $\alpha_{f}$ of the vector valued measure $\left(m, \mu_{1}, \cdots, \mu_{n}\right)$ is the area measure, [2], [8].

Functions of types BVT, ACT, and L may be discontinuous everywhere. However, they may be considered to be the respective analogues of the functions of bounded variation, the absolutely continuous functions, and the continuous functions of bounded variation in the one variable case.

${ }^{1}$ Supported by National Science Foundation Grant No. GP 03515. A detailed paper will appear in Acta Mathematica. 
2. Results and theorems. For functions of type BVT, we have the following beautiful theorem of Michael, [10], [6].

THEOREM A. If $f$ is of type BVT, and has support in a cube $Q$, then for every $\epsilon>0$ there is a continuously differentiable $g$ such that the measure of the set on which $f$ is different from $g$ is less than $\epsilon$ and $\mid \alpha_{f}(Q)$ $-\alpha_{g}(Q) \mid<\epsilon$.

An immediate consequence of this fact, which is of interest, is the following theorem.

THEOREM B. $f \in A C T$ if and only if for every $\epsilon>0$ there is a continuously differentiable $g$ such that if $E=[x: f(x) \neq g(x)]$ then $\alpha_{f}(E)<\epsilon$ and $\alpha_{g}(E)<\epsilon$.

For the case of functions of two variables, we obtained, in [7], a similar characterization of functions of type L.

THEOREM C. If $f$ is a function of type BV'T of two variables, then $f$ is of type $\mathrm{L}$ if and only if, for every $\epsilon>0$, there is a continuous $g$ such that if $E=[x: f(x) \neq g(x)]$ then $\alpha_{f}(E)<\epsilon$ and $\alpha_{g}(E)<\epsilon$.

In particular, this implies that functions of type $\mathrm{L}$ are independent of coordinates. Functions which are not of type BVT may be linearly continuous for one coordinate system but not for another one, [7].

We cannot prove Theorem $\mathrm{C}$ for $n>2$. In this paper, we are able to prove a theorem of this sort for $n$ variables, with continuity replaced by approximate continuity.

THEOREM I. If $f$ is a function of type BVT then $f$ is linearly continuous of and only if, for every $\epsilon>0$, there is an approximately continuous $g$ such that if $E=[x: f(x) \neq g(x)]$ then $\alpha_{f}(E)<\epsilon$ and $\alpha_{g}(E)<\epsilon$.

We shall not make any remarks regarding the proof of this theorem except to say that part of it involves the following fact which may be of interest in itself.

THEOREM II. If $f$ is of type BVT and approximately continuous, then it is linearly continuous.

Approximately continuous functions are taken by one-one bilipschitzian mappings into approximately continuous functions. Theorem I accordingly yields the following result.

THEOREM III. The class $\mathrm{L}$ is invariant under one-one bilipschitzian mappings.

We itemize several related facts. 
(a) For every $f \in \mathrm{L}$, there is a $g$ equivalent to $f$ which is continuous as a function of one variable along almost all lines in every direction.

(b) The integral means $\left\{f_{m}\right\}$ of $f$ converge uniformly to $g$ on almost all lines in every direction. Thus $\left\{f_{m}\right\}$ converges to $g$ everywhere, except on a set $S$ whose projection has $(n-1)$ measure zero on every hyperplane.

(c) If $M$ is any $C^{1}$ manifold then $g$ is defined $(n-1)$ almost everywhere on $M$.

3. Further remarks. We consider a metric which seems to play an important part in the classes of functions being discussed here.

For $f$ and $g$ of type BVT, let

$$
\delta(f, g)=\int \frac{|f-g|}{1+|f-g|} .
$$

For measures $\mu$ and $\nu$, let

$$
\Delta(\mu, \nu)=\sup |\mu(E)-\nu(E)|,
$$

where $E$ varies over the Borel sets, and let

$$
d(f, g)=\delta(f, g)+\Delta\left(\alpha_{f}, \alpha_{g}\right) .
$$

We list the following facts related to this metric.

(a) For functions of one variable, the spaces of absolutely continuous functions, functions of bounded variation, and continuous functions of bounded variation, are all complete.

(b) For functions of two variables, $\mathrm{L}$ is the completion of the set of continuous functions of type BVT.

(c) For functions of $n$ variables the spaces of functions of types BVT, ACT, and $\mathrm{L}$ are all complete.

(d) For functions of $n$ variables $\mathrm{L}$ is the completion of the set of approximately continuous functions of type BVT, and ACT is the completion of $C^{\prime}$ or of $C^{\infty}$.

\section{REFERENCES}

1. L. Cesari, Sulle funzioni a variazione limitata, Ann. Scuola Norm. Sup. Pisa (2), 5 (1936), 299-313.

2. - Surface area, Princeton, N. J., 1956.

3. J. W. Calkin, Functions of several variables and absolute continuity. I, Duke Math. J. 6 (1940), 170-186.

4. C. Goffman, Lower semicontinuity and area functionals. I. The nonparametric case, Rend. Circ. Mat. Palermo (2) 2 (1953), 203-235.

5. - Nonparametric surfaces given by linearly continuous functions, Acta Math. 103 (1960), 269-291. 
6. - Approximation of nonparametric surfaces of finite area, J. Math. Mech. 12 (1963), 237-246.

7. - Coordinate invariance of linear continuity, Arch. Rational Mech. Anal. 20 (1965), 153-162.

8. C. Goffman and J. Serrin, Sublinear functions of measures and variational integrals, Duke Math. J. 31 (1964), 159-178.

9. K. Krickeberg, Distributionen, Funktionen beschrankter Variation, und Lebesguescher Inhalt nichtparametrischer Flachen, Ann. Mat. Pura Appl. IV, 44 (1957), 105-133.

10. J. H. Michael, The equivalence of two areas for nonparametric discontinuous surfaces, Illinois J. Math. 7 (1963), 59-78.

11. C. B. Morrey, Functions of several variables and absolute continuity. II, Duke Math. J. 6 (1940), 187-215.

Purdue University 